\title{
Comparison of Different Forms for the "Spin" and "Orbital" Components of the Angular Momentum of Light
}

\author{
A. M. Stewart \\ Emeritus Faculty, The Australian National University, Canberra, ACT 0200, Australia \\ Correspondence should be addressed to A. M. Stewart, parbuckle.stewart@gmail.com \\ Received 5 September 2010; Accepted 3 March 2011 \\ Academic Editor: Boris Y. Zeldovich
}

Copyright ( 2011 A. M. Stewart. This is an open access article distributed under the Creative Commons Attribution License, which permits unrestricted use, distribution, and reproduction in any medium, provided the original work is properly cited.

We compare three attempts that have been made to decompose the angular momentum of the electromagnetic field into components of an "orbital" and "spin" nature. All three expressions are different, and there seems to be no reason to prefer one to another. It appears, on the basis of classical electrodynamics, that there is no unique way of decomposing the angular momentum of the electromagnetic field into orbital and spin components, even in a fixed inertial frame.

\section{Introduction}

The total angular momentum $\mathbf{J}$ of the electromagnetic field is given (in SI units) [1] by

$$
\mathbf{J}(t)=\varepsilon_{0} \int d^{3} x \mathbf{x} \times[\mathbf{E}(\mathbf{x}, t) \times \mathbf{B}(\mathbf{x}, t)]
$$

Henceforth, we will suppress the time coordinate $t$ of the fields, all of which depend on time, and also the $\varepsilon_{0}$ factor.

There has been debate for a long time over whether the total angular moment $\mathbf{J}$ of the electromagnetic field can be decomposed into an orbital part $\mathbf{L}$ and a spin part $\mathbf{S}$ so that

$$
\mathbf{J}=\mathbf{L}+\mathbf{S} \text {. }
$$

Some authors [2] argue that on the basis of the first principles it is not possible to do this; others [3-5] show that forms can be demonstrated that appear to be, at least algebraically, of a spin and orbital nature.

By means of partial integration Ohanian [4] effected a decomposition with $\mathbf{J}=\mathbf{L}^{\prime}+\mathbf{S}^{\prime}+\mathbf{J}_{b}^{\prime}$,

$$
\begin{gathered}
\mathbf{S}^{\prime}=\int d^{3} x \mathbf{E}(\mathbf{x}) \times \mathbf{A}(\mathbf{x}), \\
\mathbf{L}^{\prime}=\sum_{i} \int d^{3} x E^{i}(\mathbf{x})\left(\mathbf{x} \times \nabla_{x}\right) A^{i}(\mathbf{x}),
\end{gathered}
$$

where $\mathbf{A}$ is the vector potential $\left\{\mathbf{B}(\mathbf{x})=\nabla_{x} \times \mathbf{A}(\mathbf{x})\right\}$ and $\nabla_{x}$ is the gradient operator that operates on functions of $\mathbf{x}$. Ohanian assumed that the electric charge density $\rho$ was zero and deemed (3) and (4) to be the spin and orbital components, respectively, of the electromagnetic field on the basis that the integrand of (3) was not explicitly linear in the $\mathbf{x}$ coordinate whereas the integrand of (4) was. When the charge density is not zero, a bound term $\mathbf{J}_{b}^{\prime}$, considered also to be of an orbital nature

$$
\mathbf{J}_{b}^{\prime}=\int d^{3} x \mathbf{x} \times \mathbf{A}(\mathbf{x}) \nabla_{x} \cdot \mathbf{E}(\mathbf{x})=\int d^{3} x \mathbf{x} \times \mathbf{A}(\mathbf{x}) \rho(\mathbf{x}),
$$

is obtained on whose form all writers agree [6]. Although the sum of (3) and (4) and (5) $\mathbf{J}=\mathbf{L}^{\prime}+\mathbf{S}^{\prime}+\mathbf{J}_{b}^{\prime}$ is gauge invariant, the individual terms are not and so have no physical interpretation until the gauge of the vector potential is fixed completely.

Cohen-Tannoudji et al. [3] used the Coulomb (or transverse) gauge, defined by the gauge condition $\nabla \cdot \mathbf{A}_{t}=0$, which gives

$$
\begin{gathered}
\mathbf{S}^{\prime \prime}=\int d^{3} x \mathbf{E}(\mathbf{x}) \times \mathbf{A}_{t}(\mathbf{x}), \\
\mathbf{L}^{\prime \prime}=\sum_{i} \int d^{3} x E^{i}(\mathbf{x})\left(\mathbf{x} \times \nabla_{x}\right) A_{t}^{i}(\mathbf{x}), \\
\mathbf{J}_{b}^{\prime \prime}=\int d^{3} x \rho(\mathbf{x}) \mathbf{x} \times \mathbf{A}_{t}(\mathbf{x}) .
\end{gathered}
$$


Since the Coulomb gauge is a completely fixed gauge and has no remaining gauge arbitrariness, (6), (7), and (8) are individually well defined with $\mathbf{J}=\mathbf{L}^{\prime \prime}+\mathbf{S}^{\prime \prime}+\mathbf{J}_{b}^{\prime \prime}$.

Cohen-Tannoudji et al. [3] went further and expanded the electric field $\mathbf{E}$ into transverse $\mathbf{E}_{t}=-\dot{\mathbf{A}}_{t}=-\partial \mathbf{A}_{t} / \partial t$ and longitudinal $\mathbf{E}_{l}=-\nabla \phi$ parts, where $\phi$ is the scalar potential with $\mathbf{E}=\mathbf{E}_{t}+\mathbf{E}_{l}$, to get

$$
\begin{gathered}
\mathbf{S}^{\prime \prime \prime}=\int d^{3} x \mathbf{E}_{t}(\mathbf{x}) \times \mathbf{A}_{t}(\mathbf{x})=-\int d^{3} x \dot{\mathbf{A}}_{t}(\mathbf{x}) \times \mathbf{A}_{t}(\mathbf{x}), \\
\mathbf{L}^{\prime \prime \prime}=\sum_{i} \int d^{3} x E_{t}^{i}(\mathbf{x})\left(\mathbf{x} \times \nabla_{x}\right) A_{t}^{i}(\mathbf{x}) \\
=-\sum_{i} \int d^{3} x \dot{A}_{t}^{i}(\mathbf{x})\left(\mathbf{x} \times \nabla_{x}\right) A_{t}^{i}(\mathbf{x}) .
\end{gathered}
$$

The bound component remains the same as (8). It will be shown in Section 2 that the terms that involve the scalar potential in (6) and (7) cancel so that $\mathbf{J}=\mathbf{L}^{\prime \prime}+\mathbf{S}^{\prime \prime}+\mathbf{J}_{b}^{\prime \prime}$ in (6), (7), and (8) and $\mathbf{J}=\mathbf{L}^{\prime \prime \prime}+\mathbf{S}^{\prime \prime \prime}+\mathbf{J}_{b}^{\prime \prime}$ in (9), (10), and (8) but $\mathbf{S}^{\prime \prime}$ in (6) differs from $S^{\prime \prime \prime}$ in (9) and $\mathbf{L}^{\prime \prime}$ in (7) differs from $\mathbf{L}^{\prime \prime \prime}$ in (10). The forms of (9) and (10) have also been used by van Enk and Nienhuis [5]. The general explicit form for $\mathbf{A}_{t}$, given in (13), was not specified by these writers.

On the other hand, Stewart [7] found a decomposition $\mathbf{J}=\mathbf{L}^{\prime \prime \prime \prime}+\mathbf{S}^{\prime \prime \prime \prime}+\mathbf{J}_{b}^{\prime \prime}$ from decomposing the electric field by the Helmholtz theorem [8]:

$$
\begin{gathered}
\mathbf{S}^{\prime \prime \prime \prime}=\frac{1}{4 \pi} \int d^{3} x \int d^{3} y \frac{\mathbf{B}(\mathbf{x}) \times \dot{\mathbf{B}}(\mathbf{y})}{|\mathbf{x}-\mathbf{y}|} \\
\mathbf{L}^{\prime \prime \prime \prime}=\frac{1}{4 \pi} \int d^{3} x \int d^{3} y \mathbf{B}(\mathbf{x}) \cdot \dot{\mathbf{B}}(\mathbf{y}) \frac{\mathbf{x} \times \mathbf{y}}{|\mathbf{x}-\mathbf{y}|^{3}} .
\end{gathered}
$$

This decomposition uses the $\mathbf{E}$ and $\mathbf{B}$ fields throughout so no issues of gauge arbitrariness arise. Nor is there any complication that arises from using the longitudinal and transverse components of the fields, as $\mathbf{B}$ is entirely transverse. The expression for $\mathbf{J}_{b}^{\prime \prime}$ was again given by (8) [6]. Equations (8), (11), and (12) have been used elsewhere [9] to show that the electromagnetic field makes zero contribution to the angular momentum of the physical electron described by the Lagrangian of quantum electrodynamics and to resolve the paradox concerning the angular momentum of a plane electromagnetic wave [10]. Application has also been made to paraxial rays [11] where it has been shown that the same results are obtained from (11) and (12) as from (9) and (10).

The question addressed in this paper is how (11) and (12) are related to (9) and (10) when the relation below (13) [12, 13] that expresses the vector potential of the Coulomb gauge explicitly in terms of the instantaneous magnetic field is used:

$$
\begin{aligned}
\mathbf{A}_{t}(\mathbf{x}) & =\nabla_{x} \times \int d^{3} y \frac{\mathbf{B}(\mathbf{y})}{4 \pi|\mathbf{x}-\mathbf{y}|} \\
& =-\int \frac{d^{3} y}{4 \pi} \mathbf{B}(\mathbf{y}) \times \nabla_{x} \frac{1}{|\mathbf{x}-\mathbf{y}|} .
\end{aligned}
$$

In Section 2 we summarise the derivation [3] leading from (1) to (9) and (10). In Section 3 we obtain an expression for
$\mathbf{S}^{\prime \prime \prime}$ of (9) and $\mathbf{L}^{\prime \prime \prime}$ of (10) using (13) and demonstrate that $\mathbf{L}^{\prime \prime \prime}+\mathbf{S}^{\prime \prime \prime}=\mathbf{L}^{\prime \prime \prime \prime}+\mathbf{S}^{\prime \prime \prime \prime}$. Section 4 summarises the conclusion of the paper that there is, on the basis of classical physics, no unique decomposition of the angular momentum of the electromagnetic field into spin and orbital components, even in a fixed inertial frame.

\section{Derivation of the Standard Version of the Angular Momentum Decomposition}

To obtain (3) and (4) from (1) we consider the component of the cross product

$$
\mathbf{E} \times\left.\left(\nabla_{x} \times \mathbf{A}\right)\right|^{i}=\varepsilon^{i j k} \varepsilon^{k l m} E^{j} \frac{\partial A^{m}}{\partial x^{l}} .
$$

By multiplying the Levi-Civita symbols we find [4]

$$
\mathbf{E} \times\left(\nabla_{x} \times \mathbf{A}\right)=E^{n} \nabla_{x} A^{n}-\left(\mathbf{E} \cdot \nabla_{x}\right) \mathbf{A} .
$$

Next, consider the identity

$$
\frac{\partial}{\partial x^{m}}\left(x^{j} E^{m} A^{k}\right)=\delta_{j, m} E^{m} A^{k}+x^{j}\left(A^{k} \frac{\partial E^{m}}{\partial x^{m}}+E^{m} \frac{\partial A^{k}}{\partial x^{m}}\right)
$$

and integrate this over $x^{m}$ from minus infinity to plus infinity. The left-hand side vanishes because the integrand is zero at those points. Integrate over the other two components of $\mathbf{x}$ to produce a volume integral over all space of $\mathrm{d}^{3} x$ then sum over $m$. The result, when multiplied by $\varepsilon^{i j k}$ and integrated, substituted into the angular momentum obtained from (15), and specialised to the Coulomb gauge, is

$$
\mathbf{J}=\int d^{3} x\left\{\mathbf{x} \times \mathbf{A}_{t}\left(\nabla_{x} \cdot \mathbf{E}\right)+\mathbf{E} \times \mathbf{A}_{t}+E^{n}\left(\mathbf{x} \times \nabla_{x}\right) A_{t}^{n}\right\} .
$$

This reproduces (3), (4), and (5) for general gauge and (6), (7), and (8) for the Coulomb gauge.

The next step is to decompose the electric field into its longitudinal $-\nabla \phi$ and transverse $\mathbf{E}_{t}$ components. Since the divergence of a transverse vector field is zero, the first term in (17) is unchanged from (8). The term associated with the potential in the third term of (17) is

$$
\left.\Delta J^{3}\right|^{i}=-\sum_{j, k, n} \int d^{3} x \varepsilon^{i j k} \frac{\partial \phi}{\partial x^{n}} x^{j} \frac{\partial A_{t}^{n}}{\partial x^{k}} .
$$

Consider the identity

$$
\frac{\partial}{\partial x^{k}}\left(x^{j} A_{t}^{n} \frac{\partial \phi}{\partial x^{n}}\right)=\delta_{j, k} A_{t}^{n} \frac{\partial \phi}{\partial x^{n}}+x^{j}\left(\frac{\partial A_{t}^{n}}{\partial x^{k}} \frac{\partial \phi}{\partial x^{n}}+A_{t}^{n} \frac{\partial^{2} \phi}{\partial x^{k} \partial x^{n}}\right) .
$$

When (19) is put into (18), the term coming from the first term of the right-hand side of (19) vanishes because of the product of the Kronecker delta and the Levi Civita functions, and we get

$$
\left.\Delta J^{3}\right|^{i}=\sum_{j, k, n} \int d^{3} x \varepsilon^{i j k} x^{j} A_{t}^{n} \frac{\partial^{2} \phi}{\partial x^{k} \partial x^{n}}
$$


From the identity

$$
\frac{\partial}{\partial x^{n}}\left(x^{j} A_{t}^{n} \frac{\partial \phi}{\partial x^{k}}\right)=\delta_{j, n} A_{t}^{n} \frac{\partial \phi}{\partial x^{k}}+x^{j}\left(\frac{\partial A_{t}^{n}}{\partial x^{n}} \frac{\partial \phi}{\partial x^{k}}+A_{t}^{n} \frac{\partial^{2} \phi}{\partial x^{k} \partial x^{n}}\right)
$$

and noting that $\nabla \cdot \mathbf{A}_{t}=0$, we find

$$
\left.\Delta J^{3}\right|^{i}=-\sum_{j, k, n} \int d^{3} x \varepsilon^{i j k} \delta_{j, n} A_{t}^{n} \frac{\partial \phi}{\partial x^{k}}=-\sum_{j, k} \int d^{3} x \varepsilon^{i j k} A_{t}^{j} \frac{\partial \phi}{\partial x^{k}} .
$$

The angular momentum of the second term of (17) arising from the potential is

$$
\left.\Delta J^{2}\right|^{i}=-\sum_{j, k} \int d^{3} x \varepsilon^{i j k} \frac{\partial \phi}{\partial x^{j}} A_{t}^{k}
$$

Noting the order of the indices, (22) and (23) sum to zero; so we find that the contribution of the longitudinal electric field to the total angular momentum is zero, so $\mathbf{J}=\mathbf{L}^{\prime \prime \prime}+$ $\mathbf{S}^{\prime \prime \prime}+\mathbf{J}_{b}^{\prime \prime}$ with the components $\mathbf{L}^{\prime \prime \prime}$ and $\mathbf{S}^{\prime \prime \prime}$ given by (9) and (10). We see already that the angular momentum of the electromagnetic field can be expressed in two different ways, as $\mathbf{J}=\mathbf{L}^{\prime \prime \prime}+\mathbf{S}^{\prime \prime \prime}+\mathbf{J}_{b}^{\prime \prime}$ given by (6), (7), and (8) and as $\mathbf{J}=\mathbf{L}^{\prime \prime}+\mathbf{S}^{\prime \prime}+\mathbf{J}_{b}^{\prime \prime}$ given by (9), (10), and (8).

\section{Confirmation of the New Version of the Angular Momentum Decomposition}

In this section of the paper we substitute the expression for the vector potential of the Coulomb gauge (13) into (9) and (10) in order to find how (9) and (10) are related to (11) and (12). We will find that the sum of (9) and (10) is equal to the sum of (11) and (12). First we examine $\mathbf{L}^{\prime \prime \prime}$ of (10). This may be expressed as

$$
\begin{aligned}
\mathbf{L}^{\prime \prime \prime} & =-\int d^{3} x \mathbf{x} \times\left.\nabla_{x}\left[\dot{\mathbf{A}}_{t}(\mathbf{z}) \cdot \mathbf{A}_{t}(\mathbf{x})\right]\right|_{\mathbf{z}=\mathbf{x}} \\
& =-\int d^{3} x \mathbf{x} \times\left\{\dot{\mathbf{A}}_{t}(\mathbf{x}) \times \mathbf{B}(\mathbf{x})+\left[\dot{\mathbf{A}}_{t}(\mathbf{x}) \cdot \nabla_{\mathbf{x}}\right] \mathbf{A}_{t}(\mathbf{x})\right\}
\end{aligned}
$$

Using (13), the first term of (24) comes to

$$
\mathbf{L}^{\prime \prime \prime 1}=-\int d^{3} x \int \frac{d^{3} y}{4 \pi} \mathbf{x} \times\left\{\mathbf{B}(\mathbf{x}) \times\left[\dot{\mathbf{B}}(\mathbf{y}) \times \nabla_{x} \frac{1}{|\mathbf{x}-\mathbf{y}|}\right]\right\},
$$

and, by multiplying out the triple vector product, this becomes

$$
\begin{aligned}
\mathbf{L}^{\prime \prime \prime}=-\int d^{3} x \int \frac{d^{3} y}{4 \pi} \mathbf{x} \times & \left\{\dot{\mathbf{B}}(\mathbf{y})\left[\mathbf{B}(\mathbf{x}) \cdot \nabla_{x} \frac{1}{|\mathbf{x}-\mathbf{y}|}\right]\right. \\
& \left.-\nabla_{x} \frac{1}{|\mathbf{x}-\mathbf{y}|}[\dot{\mathbf{B}}(\mathbf{y}) \cdot \mathbf{B}(\mathbf{x})]\right\} .
\end{aligned}
$$

When the gradient in the second term of $(26), \mathbf{L}^{\prime \prime \prime 12}$, is expressed explicitly, the term becomes

$$
\mathbf{L}^{\prime \prime \prime 12}=\frac{1}{4 \pi} \int d^{3} x \int d^{3} y \dot{\mathbf{B}}(\mathbf{y}) \cdot \mathbf{B}(\mathbf{x}) \frac{\mathbf{x} \times \mathbf{y}}{|\mathbf{x}-\mathbf{y}|^{3}} .
$$

The first term in (26) is expressed in components as

$\left.\mathbf{L}^{\prime \prime \prime 11}\right|^{i}=-\sum_{j, k, m} \int d^{3} x \int \frac{d^{3} y}{4 \pi} \varepsilon^{i j k} x^{j} \dot{B}^{k}(\mathbf{y}) B^{m}(\mathbf{x}) \frac{\partial}{\partial x^{m}} \frac{1}{|\mathbf{x}-\mathbf{y}|}$.

By considering the identity

$$
\begin{aligned}
\frac{\partial}{\partial x^{m}}\left[\frac{\dot{B}^{k}(\mathbf{y}) x^{j} B^{m}(\mathbf{x})}{|\mathbf{x}-\mathbf{y}|}\right] & \\
= & \dot{B}^{k}(\mathbf{y})\left\{\delta_{j, m} \frac{B^{m}(\mathbf{x})}{|\mathbf{x}-\mathbf{y}|}\right. \\
& \left.+x^{j}\left[B^{m}(\mathbf{x}) \frac{\partial}{\partial x^{m}} \frac{1}{|\mathbf{x}-\mathbf{y}|}+\frac{1}{|\mathbf{x}-\mathbf{y}|} \frac{\partial B^{m}(\mathbf{x})}{\partial x^{m}}\right]\right\},
\end{aligned}
$$

we obtain, with $\nabla \cdot \mathbf{B}=0$,

$$
\left.\mathbf{L}^{\prime \prime \prime 11}\right|^{i}=\sum_{j, k, m} \int d^{3} x \int \frac{d^{3} y}{4 \pi} \varepsilon^{i j k} \dot{B}^{k}(\mathbf{y}) \frac{\delta_{j m} B^{m}(\mathbf{x})}{|\mathbf{x}-\mathbf{y}|}
$$

or

$$
\mathbf{L}^{\prime \prime \prime 11}=\frac{1}{4 \pi} \int d^{3} x \int d^{3} y \frac{\mathbf{B}(\mathbf{x}) \times \dot{\mathbf{B}}(\mathbf{y})}{|\mathbf{x}-\mathbf{y}|}
$$

The second term $\mathbf{L}^{\prime \prime \prime 2}$ of (24) is simplified by writing it in components and using the relation

$$
\frac{\partial}{\partial x^{m}}\left(x^{j} \dot{A}_{t}^{m} A_{t}^{k}\right)=\delta_{j m} \dot{A}_{t}^{m} A_{t}^{k}+x^{j}\left(\frac{\partial \dot{A}_{t}^{m}}{\partial x^{m}} A_{t}^{k}+\dot{A}_{t}^{m} \frac{\partial A_{t}^{k}}{\partial x^{m}}\right)
$$

with the gauge condition $\nabla \cdot \mathbf{A}_{t}=0$ to get

$$
\mathbf{L}^{\prime \prime \prime 2}=\int d^{3} x \dot{\mathbf{A}}_{t}(\mathbf{x}) \times \mathbf{A}_{t}(\mathbf{x})
$$

Accordingly, $\mathbf{L}^{\prime \prime \prime}=\mathbf{L}^{\prime \prime \prime 11}+\mathbf{L}^{\prime \prime \prime 12}+\mathbf{L}^{\prime \prime \prime 2}$ and

$$
\begin{aligned}
\mathbf{L}^{\prime \prime \prime}= & \frac{1}{4 \pi} \int d^{3} x \int d^{3} y \frac{\mathbf{B}(\mathbf{x}) \times \dot{\mathbf{B}}(\mathbf{y})}{|\mathbf{x}-\mathbf{y}|} \\
& +\frac{1}{4 \pi} \int d^{3} x \int d^{3} y \dot{\mathbf{B}}(\mathbf{y}) \cdot \mathbf{B}(\mathbf{x}) \frac{\mathbf{x} \times \mathbf{y}}{|\mathbf{x}-\mathbf{y}|^{3}} \\
& +\int d^{3} x \dot{\mathbf{A}}_{t}(\mathbf{x}) \times \mathbf{A}_{t}(\mathbf{x}) .
\end{aligned}
$$

Since $S^{\prime \prime \prime}$ of (9) is given by the negative of the last term of (34), it follows that $\mathbf{L}^{\prime \prime \prime}+\mathbf{S}^{\prime \prime \prime}=\mathbf{L}^{\prime \prime \prime}+\mathbf{S}^{\prime \prime \prime}$, confirming the validity of (11) and (12). 


\section{Conclusion}

In this paper we have compared three different expressions for the angular momentum of the electromagnetic field; they are

$$
\begin{aligned}
\mathbf{J}= & \int d^{3} x\left\{\mathbf{x} \times \mathbf{A}_{t} \rho(\mathbf{x})+\varepsilon_{0} \mathbf{E} \times \mathbf{A}_{t}+\varepsilon_{0} E^{n}\left(\mathbf{x} \times \nabla_{x}\right) A_{t}^{n}\right\}, \\
\mathbf{J}= & \int d^{3} x\left\{\mathbf{x} \times \mathbf{A}_{t} \rho(\mathbf{x})+\varepsilon_{0} \mathbf{E}_{t} \times \mathbf{A}_{t}+\varepsilon_{0} E_{t}^{n}\left(\mathbf{x} \times \nabla_{x}\right) A_{t}^{n}\right\}, \\
\mathbf{J}= & \int d^{3} x \mathbf{x} \times \mathbf{A}_{t}(\mathbf{x}) \rho(\mathbf{x})+\frac{\varepsilon_{0}}{4 \pi} \int d^{3} x \int d^{3} y \frac{\mathbf{B}(\mathbf{x}) \times \dot{\mathbf{B}}(\mathbf{y})}{|\mathbf{x}-\mathbf{y}|} \\
& +\frac{\varepsilon_{0}}{4 \pi} \int d^{3} x \int d^{3} y \mathbf{B}(\mathbf{x}) \cdot \dot{\mathbf{B}}(\mathbf{y}) \frac{\mathbf{x} \times \mathbf{y}}{|\mathbf{x}-\mathbf{y}|^{3}},
\end{aligned}
$$

where the vector potential of the Coulomb gauge $\mathbf{A}_{t}$ is given by (13) and the $\varepsilon_{0}$ factor has been restored. The expressions all have the algebraic form of the sum of a spin and an orbital components, and each component of all three expressions is gauge invariant. All three expressions are different, and, on the basis of classical electrodynamics, there seems to be no reason to prefer one to another. The last form (37) [7] has the pedagogical advantage of being derived entirely in terms of fields through the Helmholtz theorem; issues of gauge arbitrariness do not have to be addressed. It appears that there is no unique way of decomposing the angular momentum of the classical electromagnetic field into orbital and spin components, even in an inertial frame.

\section{References}

[1] J. D. Jackson, Classical Electrodynamics, John Wiley \& Sons, New York, NY, USA, 3rd edition, 1999.

[2] W. Heitler, The Quantum Theory of Radiation, Clarendon Press, Oxford, UK, 3rd edition, 1954.

[3] C. Cohen-Tannoudji, J. Dupont-Roc, and G. Gilbert, Photons and Atoms, John Wiley \& Sons, New York, NY, USA, 1989.

[4] H. C. Ohanian, "What is spin?" American Journal of Physics, vol. 54, no. 6, pp. 500-505, 1986.

[5] S. J. van Enk and G. Nienhuis, "Commutation rules and eigenvalues of spin and orbital angular momentum of radiation fields," Journal of Modern Optics, vol. 41, no. 5, pp. 963-977, 1994.

[6] A. M. Stewart, "Equivalence of two mathematical forms for the bound angular momentum of the electromagnetic field," Journal of Modern Optics, vol. 52, no. 18, pp. 2695-2698, 2005.

[7] A. M. Stewart, "Angular momentum of light," Journal of Modern Optics, vol. 52, no. 8, pp. 1145-1154, 2005.

[8] A. M. Stewart, "Longitudinal and transversecomponents of a vector field," Classical Physics. In press, http://arxiv.org/abs/ $0801.0335 \mathrm{v} 2$.

[9] A. M. Stewart, "Angular momentum of the physical electron," Canadian Journal of Physics, vol. 87, no. 2, pp. 151-152, 2009.

[10] A. M. Stewart, "Angular momentum of the electromagnetic field: the plane wave paradox resolved," European Journal of Physics, vol. 26, no. 4, pp. 635-641, 2005.
[11] A. M. Stewart, "Derivation of the paraxial form of the angular momentum of the electromagnetic field from the general form," Journal of Modern Optics, vol. 53, no. 13, pp. 19471952, 2006.

[12] A. M. Stewart, "Vector potential of the Coulomb gauge," European Journal of Physics, vol. 24, no. 5, pp. 519-524, 2003.

[13] A. M. Stewart, "Reply to Comments on 'Vector potential of the Coulomb gauge," European Journal of Physics, vol. 25, no. 2, pp. L29-L30, 2004. 

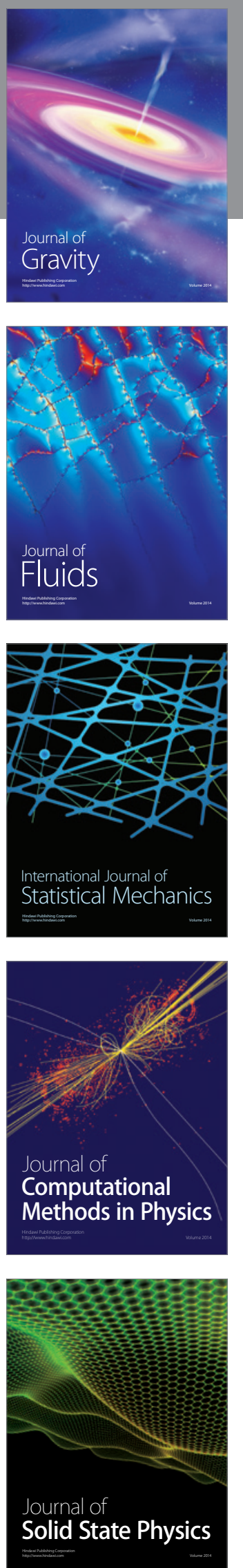

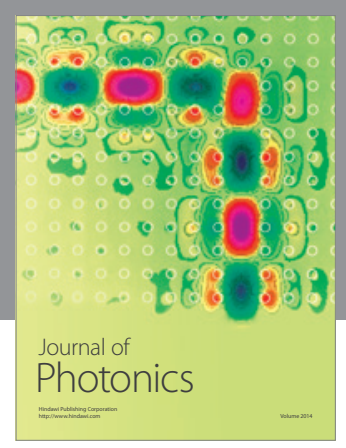

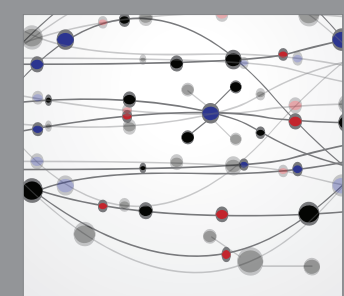

The Scientific World Journal
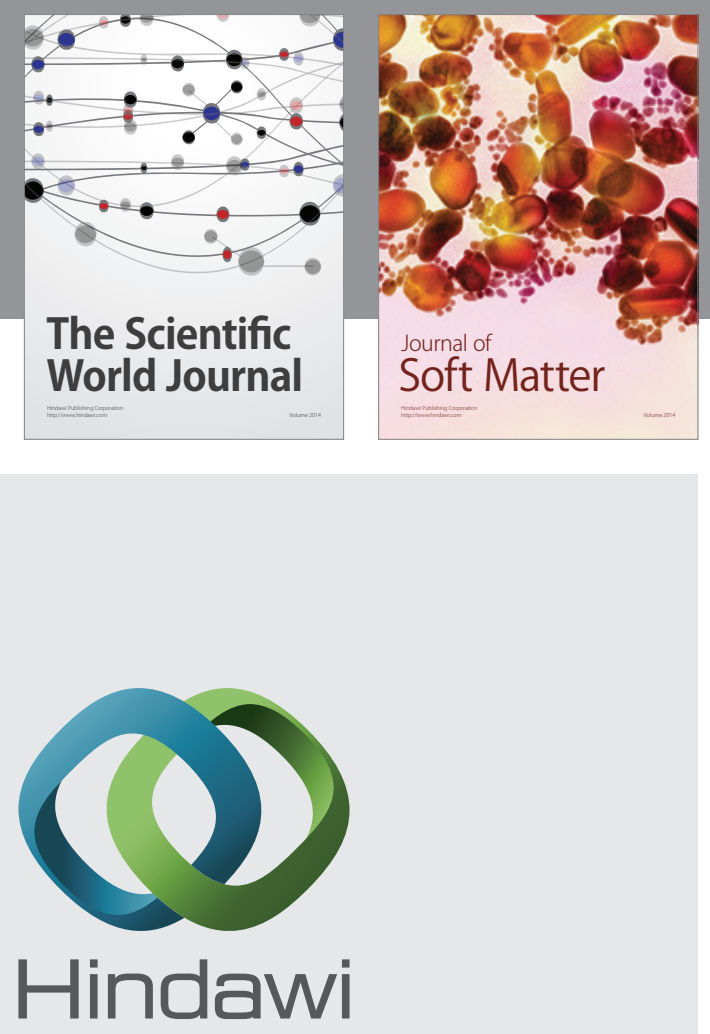

Submit your manuscripts at

http://www.hindawi.com
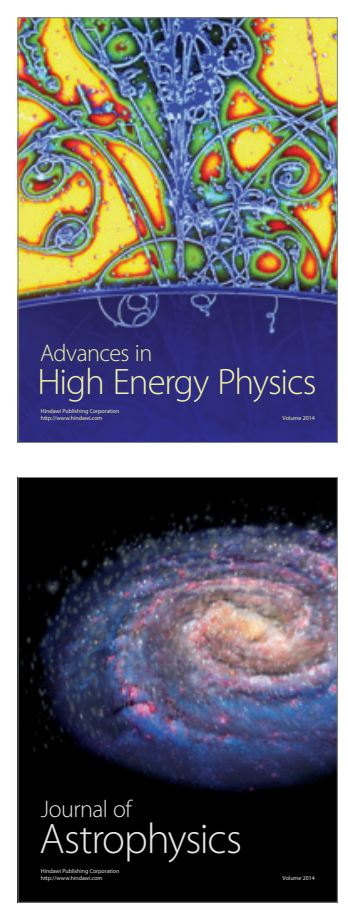
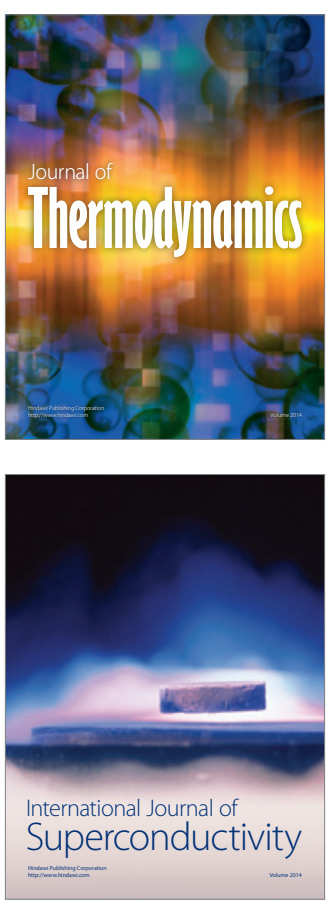
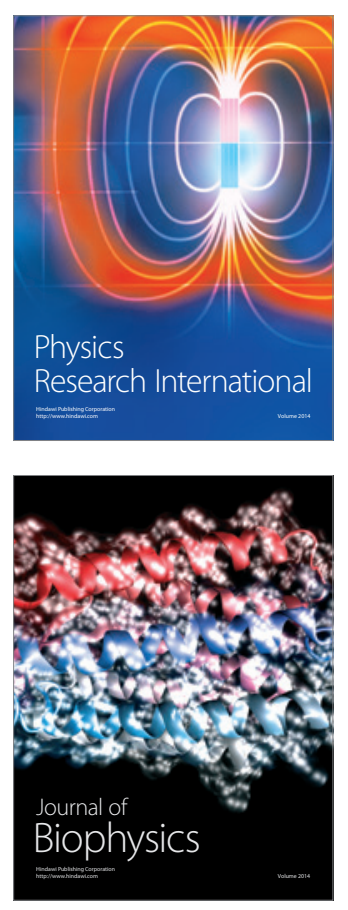
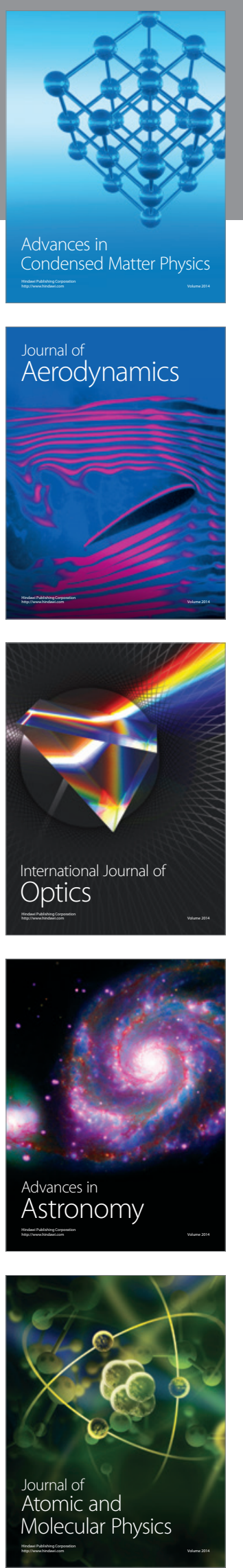